VOL. 51 (1995) [465-479]

\title{
LINEAR PARABOLIC EQUATIONS WITH VENTTSEL INITIAL BOUNDARY CONDITIONS
}

\author{
YI ZENG AND YOUSONG LUO
}

\begin{abstract}
The Schauder estimates for solutions of linear second order parabolic equations with Venttsel initial boundary conditions are proved, and existence and uniqueness of classical solutions under such an initial boundary condition are established. An application to an engineering problem is also given.
\end{abstract}

\section{INTRODUCTION}

In this paper we are concerned with problems of second order parabolic equations with initial conditions and boundary conditions of Venttsel type.

Recently, some work has been done on Venttsel boundary value problems of elliptic equations, see Luo and Trudinger $[5,6]$, and Korman [4]. Our task here is to extend the corresponding theory in the elliptic case to the parabolic case.

The motivation of such a consideration is the engineering problem of an oil well. A mathematical model of the "oil well" formulated by Cannon and Meyer [1] is included in Section 2. Instead of dealing with this particular model, we consider the general problems which are also defined in Section 2.

In Section 3, the main theorem of existence and uniqueness of solutions of the initial boundary problems is stated, and its proof follows in Section 4.

\section{THE PROBLEM}

We first introduce the problem and then give an example of the physical background of it.

2.1 Notation AND DEFinitions. Let $\Omega$ be a bounded domain in $\mathbb{R}^{n}$ with smooth boundary $\partial \Omega$. Let $\mathcal{D}$ be the domain $\mathcal{D}=\Omega \times(0, T]$ in $\mathbb{R}^{n+1}$ and $S$ be the portion of the boundary of $\mathcal{D}, S=\partial \Omega \times(0, T]$. A variable in $\mathcal{D}$ has the form

$$
P=(x, t)=\left(x_{1}, x_{2}, \cdots, x_{n}, t\right)
$$

Received 16 February 1994

Copyright Clearance Centre, Inc. Serial-fee code: 0004-9729/95 \$A2.00+0.00. 
where $x$ is called a space variable and $t$ is called a time variable. If $u$ is a function defined in $\mathcal{D}$, we denote the space derivatives and the time derivative of $u$ by

$$
D_{i} u=\frac{\partial u}{\partial x_{i}}, \quad D_{i j} u=\frac{\partial^{2} u}{\partial x_{i} \partial x_{j}}, \quad \text { and } \quad D_{t} u=\frac{\partial u}{\partial t}
$$

respectively.

A function $u$ is said to be Hölder continuous with index $0<\alpha \leqslant 1$ in $\mathcal{D}$ if for each pair of points $P=\left(x_{1}, t_{1}\right), Q=\left(x_{2}, t_{2}\right)$ in $\mathcal{D}$

$$
[u]_{\alpha}=\sup _{\mathcal{D}} \frac{|u(p)-u(Q)|}{d(P, Q)^{\alpha}}
$$

is finite, where

$$
d(P, Q)=\left(\left|x_{1}-x_{2}\right|^{2}+\left|t_{1}-t_{2}\right|\right)^{1 / 2}
$$

is the parabolic distance from $\left(x_{1}, t_{1}\right)$ to $\left(x_{2}, t_{2}\right)$. We denote the class of all Hölder continuous functions by $C^{\alpha}(\overline{\mathcal{D}})$. We use $C^{2+\alpha}(\overline{\mathcal{D}})$ to denote the class of all functions $u$ such that

$$
u, \quad D u \quad D^{2} u, \quad D_{t} u
$$

are all Hölder continuous.

Both $C^{\alpha}(\overline{\mathcal{D}})$ and $C^{2+\alpha}(\overline{\mathcal{D}})$ are Banach spaces with norms defined as following:

$$
\begin{gathered}
|u|_{\alpha ; \mathcal{D}}=|u|_{0 ; \mathcal{D}}+\left[\left.u\right|_{\alpha ; \mathcal{D}},\right. \\
|u|_{2+\alpha ; \mathcal{D}}=|u|_{\alpha ; \mathcal{D}}+|D u|_{\alpha ; \mathcal{D}}+\left|D^{2} u\right|_{\alpha ; \mathcal{D}}+\left|D_{t} u\right|_{\alpha ; \mathcal{D}}
\end{gathered}
$$

where $|u|_{0 ; \mathcal{D}}=\sup _{\mathcal{D}}|u|$.

\section{DEFINITION 2.1. An operator}

$$
L \equiv a^{i j} D_{i j}+b^{i} D_{i}+c-D_{t} \quad \text { in } \mathcal{D}
$$

is called a second order parabolic operator if the coefficient matrix $\left\{a^{i j}(x, t)\right\}$ is symmetric positive definite, that is, for some positive $\lambda(x, t), \Lambda(x, t)$

$$
\lambda|\xi|^{2} \leqslant a^{i j} \xi_{i} \xi_{j} \leqslant \Lambda|\xi|^{2} \quad \text { for all } \xi=\left(\xi_{1}, \cdots, \xi_{n}\right) \in \mathbb{R}^{n} .
$$

In order to state the Venttsel boundary condition we need to define the tangential differential operators. Let $\nu=\left(\nu^{1}, \ldots, \nu^{n}\right)$ be the unit inward normal vector field on $\partial \Omega$. Define a matrix $\left\{c^{i k}\right\}_{n \times n}$ whose entries are given by

$$
c^{i k}=\delta^{i k}-\nu^{i} \nu^{k}
$$


where $\delta^{i k}$ is the Kronnecker symbol. Then the first order tangential differential operator and the second order tangential differential operator are then defined by

$$
\partial_{i}=c^{i k} D_{k}, \quad \partial_{i j}=\partial_{i} \partial_{j}, \quad i, j=1, \cdots, n
$$

and the tangential gradient operator is defined by

$$
\partial=\left(\partial_{1}, \cdots, \partial_{n}\right) .
$$

The second order tangential derivatives so defined are not symmetric generally.

Using the above notation, we define the Venttsel boundary operator as follows:

DEFINITION 2.2. A linear differential operator

$$
l \equiv \alpha^{i j} \partial_{i j}+\beta^{i} \partial_{i}+\gamma-D_{t} \quad \text { on } S
$$

is called Venttsel if

(i) $\left\{\alpha^{i j}(x, t)\right\}$ is a non-negative definite symmetric matrix valued function, that is

$$
\alpha^{i j}(x, t) \eta_{i} \eta_{j} \geqslant 0, \quad \forall(x, t) \in S \text { and } \forall \eta \in \mathbb{R}^{n} ;
$$

(ii) The vector field $\beta=\left(\beta^{1}, \ldots, \beta^{n}\right)$ satisfies

The conditions (2.4) and (2.5) show that a Venttsel boundary operator is both parabolic and oblique.

Sometimes it is convenience for us to write the operator (2.3) as

$$
l \equiv \bar{\alpha}^{i j} D_{i j} u+\bar{\beta}^{i} D_{i} u+\gamma-D_{t}
$$

with $\bar{\alpha}^{i j}=\alpha^{k l} c^{k i} c^{l j}$ and $\bar{\beta}^{i}=\alpha^{k l} c^{k j}\left(D_{j} c^{l i}\right)+\beta^{i}$, and we observe that

$$
\overline{\boldsymbol{\alpha}}^{i j} \nu^{i}=0 \quad \forall(x, t) \in S \text { and } \forall i=1, \ldots, n .
$$

Now we are in the position to state the Venttsel initial boundary value problem of second order parabolic equations as follows:

$$
\begin{array}{ll}
L u=a^{i j} D_{i j} u+b^{i} D_{i} u+c u-D_{t} u=f & \text { in } \mathcal{D}, \\
u(x, 0)=\varphi(x) & \text { on } \bar{\Omega} \\
l u=\alpha^{i j} \partial_{i j} u+\beta^{i} D_{i} u+\gamma u-D_{t} u=g & \text { on } S .
\end{array}
$$


2.2 Hydraulic Fracturing. In the field of oil well engineering, high yield of oil in a well is the most important aspect. In order to increase the flow of oil from a reservoir into a well, "hydraulic fracturing" is often used. By mechanically fracturing the oil bearing formation near the well, narrow channels of high permeability are increased which collect the oil and carry it to the well. The following mathematical model of this situation was developed by Cannon and Meyer [1].

Consider a region $\Omega$ (the reservoir) with outer boundary $\partial \Omega$ (the reservoir boundary). If the fluid is assumed to be slightly compressible, then Darcy's law gives the following equation for the reservoir pressure $p$

$$
D_{i}\left(a(x) D_{i} p\right)-b(x) D_{t} p=F(x, t) \quad x \in \Omega, \quad t>0
$$

where $a$ and $b$ are positive functions describing the permeability of the reservoir, while $F$ is determined by the forces acting on the fluid. Let $\Gamma$ be the well boundary. Known pressure on the well boundary gives

$$
p=\phi(x, t), \quad x \in \Gamma, \quad t>0 .
$$

No flow across the reservoir boundary gives

$$
\frac{\partial p}{\partial n}=0 \quad x \in \partial \Omega, \quad t>0,
$$

where $n$ is the inward normal on $\partial \Omega$. In addition, an initial pressure distribution

$$
p=p_{0}(x), \quad x \in \Omega, \quad t=0,
$$

is provided. By assuming that $\psi=\psi(x, t)$ is a $C^{2}$ function such that

$$
\begin{aligned}
\psi & =\phi, & & x \in \Gamma, & & t>0, \\
\frac{\partial \psi}{\partial n} & =0 & & x \in \partial \Omega, & & t>0, \\
\psi & =p_{0}, & & x \in \Omega & & t=0
\end{aligned}
$$

and letting

$$
u=p-\psi,
$$

we reduce the problem $(2.10)-(2.13)$ to

$$
D_{i}\left(a D_{i}(u+\psi)\right)-b D_{t}(u+\psi)=F
$$




$$
\begin{aligned}
& u=0 \quad x \in \Gamma, \quad t>0, \\
& \frac{\partial u}{\partial n}=0 \quad x \in \partial \Omega, \quad t>0, \\
& u=0, \quad x \in \Gamma, \quad t=0 .
\end{aligned}
$$

Suppose now that $\Omega_{1}$ is a long narrow fracture crack in the reservoir outside of the well, whose centre can be approximated by a surface $S$ with its normal $\nu$ on $S$.

The local width of the fracture, measured along $\nu$, is denoted by $h(x)$, where $x \in S$. The basic assumption here is that pressure gradient across the fracture is negligible so that

$$
D u \approx \partial u
$$

where $\partial u$ is the tangential gradient of $u$.

Suppose that the permeability function $a$ is discontinuous across $S$. Then a function $u$ is a solution if it satisfies (2.17)-(2.20) away from $S$ while on $S$ continuity of pressure and flux are required, so that

$$
\begin{aligned}
\lim _{\epsilon \rightarrow 0} u(x+\epsilon \nu) & =\lim _{\epsilon \rightarrow 0} u(x-\epsilon \nu), \\
\lim _{\epsilon \rightarrow 0} a(x+\epsilon \nu) \frac{\partial u}{\partial \nu}(x+\epsilon \nu) & =\lim _{\epsilon \rightarrow 0}(x-\epsilon \nu) \frac{\partial u}{\partial \nu}(x-\epsilon \nu)
\end{aligned}
$$

where $\nu$ is the normal to $S$ at $x \in S$. Thus, in the classical sense (2.17)-(2.20) described a parabolic interface problem

$$
\begin{aligned}
D_{i}\left(a D_{i}(u+\psi)\right)-b D_{t}(u+\psi)-F=0 & \text { on } \Omega-\bar{S} \\
2 a \frac{\partial(u+\psi)}{\partial \nu}+\partial_{i}\left(a_{1} h \partial_{i}(u+\psi)\right)-b D_{t}(u+\psi)-F=0 & \text { on } S
\end{aligned}
$$

where $a_{1}$ is the permeability along the fracture $S$, and

$$
\begin{aligned}
& u=0 \quad \text { on } \Gamma \quad t>0, \\
& \frac{\partial u}{\partial n}=0 \quad \text { on } \partial \Omega \quad t>0 \\
& u=0 \quad \text { on } \Omega \quad t=0 .
\end{aligned}
$$

When $S$ coincides with the boundary $\Omega$, one can see that this is a Venttsel initial boundary value problem described in Section 2.1. 


\section{ThE MAIN THEOREM}

Theorem 3.1. For each $T$, let $\mathcal{D}=\Omega \times(0, T]$ be a $C^{2+\alpha}$ domain. Suppose $L$, $l$ are both parabolic and the coefficients $a^{i j}, b^{i}, c ; \alpha^{i j}, \beta^{i}, \gamma \in C^{\alpha}(\overline{\mathcal{D}})$. Then for each $f, g \in C^{\alpha}(\overline{\mathcal{D}})$ and $\varphi \in C^{2+\alpha}(\overline{\mathcal{D}})$, the problem (2.7)-(2.9) has a unique solution $u \in C^{2+\alpha}(\overline{\mathcal{D}})$.

The existence part of the theorem follows from the standard method of continuity, that is, we consider first the family of problems $\left(P_{\sigma}\right)$

$$
\begin{aligned}
L u & =f & & \text { in } \mathcal{D}, \\
U & =\varphi & & \text { on } \bar{\Omega}, \\
\sigma l u+(1-\sigma) l^{\prime} u & =\sigma g & & \text { on } S
\end{aligned}
$$

with $\sigma \in[0,1]$, and $l^{\prime}$ defined by

$$
l^{\prime} u=\Delta_{\partial \Omega} u-D_{t} u
$$

where $\Delta_{\partial \Omega}=\partial_{i i} u$ is the Laplace-Beltrami operator. The method of continuity (Theorem 5.2 of [3]) says that if

(i) the solutions $u_{\sigma}$ of $\left(P_{\sigma}\right)$ satisfy

$$
\left|u_{\sigma}\right|_{2+\alpha ; \mathcal{D}} \leqslant C
$$

for a constant $C$ which is independent of $u_{\sigma}$,

(ii) the problem $\left(P_{0}\right)$

$$
\begin{aligned}
l u=f & \text { in } \mathcal{D}, \\
u=\varphi & \text { on } \bar{\Omega}, \\
l^{\prime} u=0 & \text { on } S
\end{aligned}
$$

is solvable in $C^{2+\alpha}$,

then the problem (2.7)-(2.9) is also solvable in $C^{2+\alpha}$.

We shall leave the a priori estimates (3.4) to the sequel, and give the solution of $\left(P_{0}\right)$ here.

Let $\left\{\mathcal{N}_{i}\right\}_{i=1}^{k}$ be a finite covering for $\partial \Omega$, that is, each $\mathcal{N}_{i}$ is open and $U \mathcal{N}_{i} \supset \partial \Omega$. Let $\left\{\sigma_{i}\right\}_{i=1}^{k}$ be the partition of unity relative to $\left\{\mathcal{N}_{i}\right\}_{i=1}^{k}$, that is, $\operatorname{supp} \sigma_{i} \subset \mathcal{N}_{i}, \sigma_{i} \geqslant 0$ and $\sum_{i=1}^{k} \sigma_{i} \equiv 1$. We denote $\mathcal{N}_{i} \cap \bar{\Omega}$ by $\Omega_{i}$. Then there is a diffeomorphism $\psi_{i}: \mathcal{N}_{i} \rightarrow \mathbb{R}^{n}$, 
such that $\psi_{i}\left(\Omega_{i}\right)=B^{+}, \psi_{i}\left(\partial \Omega \cap \mathcal{N}_{i}\right)=B^{0}$, where $B^{+}=B^{+}(0)=\left\{y \in \mathbb{R}^{n}:|y|<\right.$ $\left.1, y_{n} \geqslant 0\right\}$ and $B^{0}=B^{0}(0)=\left\{y \in \mathbb{R}^{n}:|y|<1, y_{n}=0\right\}$. Then the Cauchy problem

$$
\begin{array}{rlrl}
\Delta u-D_{t} u= & =0 & & \text { in } B^{0} \times(0, T] \\
u=\hat{\varphi} & & \text { on } B^{0}
\end{array}
$$

has a solution $\widehat{u}_{i}$ by the classical theorem for heat equations. Now we define

$$
u=\sum_{i=1}^{k} u_{i},
$$

where $u_{i}=\widehat{u}_{i} \circ \psi_{i}$. It follows that $u$ is a solution of

$$
\begin{aligned}
\Delta_{\theta \Omega} u-D_{t} u=0 & \text { on } S, \\
u=\varphi & \text { on } \partial \Omega .
\end{aligned}
$$

By using this solution as the boundary data and solving the resulting first initial boundary problem of $L u=f$, we obtain a solution of the problem $\left(P_{0}\right)$.

Therefore the whole problem is reduced to the a priori estimate (3.4).

The uniqueness part of the theorem is an immediate consequence of the maximum principle in the following section.

\section{A priori ESTIMATE}

4.1 MAXIMUM PRINCIPLE. The maximum principle provides the earliest and simplest a priori estimate of a solution. It is of considerable interest that all the estimates can be derived entirely from comparison arguments based on the maximum principle. Therefore, we first develop a maximum principle for the initial boundary value problem (2.7)-(2.9).

The following lemma from [5] is fundamental to Venttsel boundary conditions. We provide a complete proof here.

Lemma 4.1. Suppose that $l$ is Ventsel. If $\left(x_{0}, t_{0}\right) \in S$ is a maximum point of some function $u \in C^{2}(\overline{\mathcal{D}})$, then at $\left(x_{0}, t_{0}\right)$, we have $\left\{\partial_{i j} u\right\} \leqslant 0, D_{\nu} u \leqslant 0, \partial u=0$, so that

$$
\alpha^{i j} \partial_{i j} u+\beta_{i} D_{i} u \leqslant 0
$$

Proof: We locally flatten the boundary in the following manner. For any point $\left(x_{0}, t_{0}\right) \in S$, we can always find a neighbourhood $\mathcal{N}$ of $\left(x_{0}, t_{0}\right)$ and a diffeomorphism $\Psi: \mathcal{N} \rightarrow \mathbb{R}^{n+1}$ such that

$$
\Psi(\mathcal{N} \cap \mathcal{D})=B^{+} \times(-1,1), \quad \Psi(\mathcal{N} \cap S)=B^{0} \times(-1,1)
$$


and

$$
\Psi\left(x_{0}, t_{0}\right)=(0,0)
$$

where $B^{+}$and $B^{0}$ are defined as before. For each continuous function $v$ in $\mathcal{N} \cap \overline{\mathcal{D}}$, we denote $\bar{v}=v \circ \Psi^{-1}$ which then belongs to $C\left(B^{+} \cup B^{0}\right)$. Under the diffeomorphism $\Psi$, we have

$$
\begin{aligned}
\partial_{i} u & =c^{i k} \frac{\partial \Psi_{\sigma}}{\partial x_{k}} D_{\sigma} \bar{u} \\
D_{\nu} u & =\nu_{k} \frac{\partial \Psi_{\sigma}}{\partial x_{k}} D_{\sigma} \bar{u}
\end{aligned}
$$

and

$$
\begin{aligned}
\partial_{i} \partial_{j} u=c^{i k} c^{j l} & \frac{\partial \Psi_{\sigma}}{\partial x_{k}} \frac{\partial \Psi_{\tau}}{\partial x_{l}} D_{\sigma \tau} \bar{u}+c^{i k} c^{j l} \frac{\partial^{2} \Psi_{\sigma}}{\partial x_{k} \partial x_{l}} D_{\sigma} \bar{u} \\
& +c^{i k}\left(D_{k} c^{j l}\right) \frac{\partial \Psi_{\sigma}}{\partial x_{l}} D_{\sigma} \bar{u} .
\end{aligned}
$$

If $u$ achieves its maximum at $\left(x_{0}, t_{0}\right)$, so does $\bar{u}$ at $(0,0)$. It follows that

$$
\begin{aligned}
& D_{\sigma} \bar{u}=0, \quad \text { for } \sigma=1, \cdots, n-1 \quad \text { and } D_{n} \bar{u} \leqslant 0 \text {, } \\
& \left\{D_{\sigma \tau} \bar{u}\right\}_{\sigma, \tau=1}^{n-1} \leqslant 0 \text {. }
\end{aligned}
$$

Notice that close to $\left(x_{0}, t_{0}\right), S$ is defined by $\left\{\Psi_{n}=0\right\} \times(-1,1)$, so we obtain

$$
D \Psi_{n}=\left|D \Psi_{n}\right| \nu
$$

which implies

$$
c^{i k} \frac{\partial \Phi_{n}}{\partial x_{k}}=0 \quad \forall i=1, \cdots, n
$$

Substituting (4.5) and (4.8) into (4.2) and (4.3), we see

$$
\partial_{i} u\left(x_{0}, t_{0}\right)=0 \quad \forall i
$$

and

$$
D_{\nu} u\left(x_{0}, t_{0}\right)=\left|D \Psi_{n}\right| D_{n} \bar{u}(0,0) \leqslant 0 .
$$

Now we proceed to show that at $\left(x_{0}, t_{0}\right),\left\{\partial_{i} \partial_{j} u\right\}$ is symmetric and nonnegative, (although $\left\{\partial_{i} \partial_{j} u\right\}$ is not symmetric generally). By substituting (4.8) into (4.4) we see that the sum

$$
c^{i k} c^{j l} \frac{\partial \Psi_{\sigma}}{\partial x_{k}} \frac{\partial \Psi_{\tau}}{\partial x_{l}} D_{\sigma \tau} \bar{u}
$$


consists of only those terms for which $1 \leqslant \sigma, \tau \leqslant n-1$. It follows that

$$
\begin{aligned}
c^{i k} c^{j l} & \frac{\partial^{2} \Psi_{n}}{\partial x_{k} \partial x_{l}} D_{n} \bar{u}+c^{i k}\left(D_{k} c^{j l}\right) \frac{\partial \Psi_{n}}{\partial x_{l}} D_{n} \bar{u} \\
= & \left|D \Psi_{n}\right| c^{i k} c^{j l} D_{k} \nu^{l} D_{n} \bar{u}+c^{i k} c^{j l}\left(D_{k}\left|D \Psi_{n}\right|\right) \nu^{l} D_{n} \bar{u} \\
& \quad+\left|D \Psi_{n}\right| c^{i k}\left(D_{k} c^{j l}\right) \nu^{l} D_{n} \bar{u} \\
= & 0 .
\end{aligned}
$$

Inserting (4.5), (4.6) and (4.11) into (4.4) yield

$$
\left\{\partial_{i} \partial_{j} u\left(x_{0}, t_{0}\right)\right\}=\left.\left\{c^{i k} c^{j l} \frac{\partial \Psi}{\partial x_{k}} \frac{\partial \Psi_{\tau}}{\partial x_{l}} D_{\sigma \tau} \bar{u}\right\}\right|_{(0,0)} \leqslant 0 .
$$

The inequality (4.1) is obtained by combining of (4.9), (4.10), (4.12) and the assumption on $l$.

THEOREM 4.2. Suppose that $L$ is degenerate parabolic and $l$ is degenerate oblique and degenerate parabolic. Let $u \in C^{2}(\overline{\mathcal{D}})$ satisfy

$$
L u \geqslant f, \quad \text { in } \mathcal{D} ; \quad u(x, 0)=\varphi, \quad \text { on } \bar{\Omega} ; \quad \text { lu } \geqslant g, \quad \text { on } S .
$$

We then have the estimates

$$
\sup _{\overline{\mathcal{D}}} u \leqslant C\left(\sup _{\mathcal{D}}|f|+\sup _{\overline{\mathcal{D}}}|\varphi|+\sup _{S}|g|\right)
$$

where $C=C(M, T)$ provided $\max \{|c|,|\gamma|\} \leqslant M$.

Proof: Assume $u$ attains a positive maximum in $\overline{\mathcal{D}}$. Let $v=e^{-\sigma t} u$ where $\sigma=M+1$. We then have

$$
\sup _{\mathcal{D}} u \leqslant e^{(M+1) T} \sup _{\mathcal{D}} v .
$$

We prove (4.13) in the following cases.

CASE I. If $v$ attains a maximum at $\left(x_{0}, t_{0}\right) \in \mathcal{D}$, applying the operator $L-\sigma$ to $v$ we have at $\left(x_{0}, t_{0}\right)$,

$$
\begin{aligned}
(L-\sigma) v & =a^{i j} D_{i j} e^{-\sigma t} u+b^{i} D_{i} e^{-\sigma t} u+(c-\sigma) e^{-\sigma t} u-\frac{\partial\left(e^{-\sigma t} u\right)}{\partial t} \\
& =e^{-\sigma t} L u \\
& \geqslant e^{-\sigma t} f .
\end{aligned}
$$

Since $a^{i j} D_{i j} v \leqslant 0, b_{i} D_{i} v=0$ and $D_{t} v \geqslant 0$ at $\left(x_{0}, t_{0}\right)$, we have

$$
(c-M-1) v \geqslant e^{-\sigma t} f,
$$


which implies

$$
\sup _{\overline{\mathcal{D}}} v \leqslant \sup _{\mathcal{D}}|f| .
$$

CASE II. If $\left(x_{0}, t_{0}\right) \in \bar{\Omega}$, we have

$$
\sup _{\overline{\mathcal{D}}} u \leqslant \sup _{\bar{\Omega}}|\varphi| .
$$

CASE III. If $\left(x_{0}, t_{0}\right) \in S$. We apply $(l-\sigma)$ to $v$ to get

$$
\begin{aligned}
(l-\sigma) v & =e^{-\sigma t} l u \\
& \geqslant e^{-\sigma t} g .
\end{aligned}
$$

By Lemma 4.1 we have at $\left(x_{0}, t_{0}\right)$,

$$
\alpha^{i j} \partial_{i j} v+\beta^{i} D_{i} v \leqslant 0
$$

and

$$
D_{t} v \geqslant 0
$$

so that

$$
(\gamma-\sigma) v \geqslant e^{-\sigma t} g
$$

that is,

$$
\sup _{\overline{\mathcal{D}}} v \leqslant \sup _{S}|g| .
$$

Combining the above three cases, we get

$$
\sup _{\overline{\mathcal{D}}} u \leqslant C\left(\sup _{\mathcal{D}}|f|+\sup _{\bar{\Omega}}|\varphi|+\sup _{S}|g|\right)
$$

where $C$ depends on $M$ and $T$.

Uniqueness of the solution to the Venttsel problem follows automatically from the maximum principle.

TheOREM 4.3. Let $L$ be degenerate parabolic in $\mathcal{D}$ and $l$ be degenerate parabolic and degenerate oblique. Suppose that $u$ and $v$ are functions in $C^{2}(\mathcal{D})$ satisfying $L u \geqslant L v$ in $\mathcal{D}, l u \geqslant l v$ on $S, u \leqslant v$ on $\bar{\Omega}$. Then $u \leqslant v$ in $\mathcal{D}$.

PROOF: Set $w=u-v$ in $\mathcal{D}$, then $L w=L(u-v) \geqslant 0$ in $\mathcal{D}, l w=l(u-v) \geqslant 0$ on $S$ and $u-v \leqslant 0$ on $\bar{\Omega}$. By Theorem 4.2,

$$
\sup _{\mathcal{D}} w \leqslant 0
$$

hence

$$
u \leqslant v \quad \text { in } \mathcal{D} \text {. }
$$


4.2 Schauder estimates. In addition to the notation introduced in Section 2 we adopt some more standard notation for Hölder norms in a parabolic problem. (See $[\mathbf{3}, \mathbf{2}]$.)

For $P=(x, t), Q=(y, s) \in \mathcal{D}$ we denote

$$
\begin{gathered}
d=d_{P}=\operatorname{dist}(P, \bar{\Omega}+S), \\
d_{P Q}=\min \left\{d_{P}, d_{Q}\right\} .
\end{gathered}
$$

and if $\Gamma$ is a portion of the boundary manifold $\bar{\Omega}+S$ we denote

$$
\begin{aligned}
\bar{d}=\bar{d}_{P} & =\operatorname{dist}(P,(\bar{\Omega}+S) \backslash \Gamma), \\
\bar{d}_{P Q} & =\min \left\{\bar{d}_{P}, \bar{d}_{Q}\right\} .
\end{aligned}
$$

A function $u$ is said to belong to the class $C^{2+\alpha}(\mathcal{D})$ if the following interior Höider norm is finite:

$$
|u|_{2+\alpha}^{*}=|u|_{\alpha}^{*}+|d D u|_{\alpha}^{*}+\left|d^{2} D^{2} u\right|_{\alpha}^{*}+\left|d^{2} D_{t} u\right|_{\alpha}^{*}
$$

where

$$
|v|_{\alpha}^{*}=|v|_{0}+[v]_{\alpha}^{*}
$$

and

$$
[v]_{\alpha}^{*}=\sup _{\mathcal{D}} d_{P Q}^{\alpha} \frac{|v(P)-v(Q)|}{d(p, Q)^{\alpha}}
$$

The class of functions $u$ which are of $C^{2+\alpha}$ not only in $\mathcal{D}$ but also up to $\Gamma$ is denoted by $C^{2+\alpha}(\mathcal{D} \cup \Gamma)$, and its norm is denoted by $|u|_{2+\alpha, \mathcal{D} \cup \Gamma}^{*}$. This norm is defined also by (4.14) except that $d$ and $d_{P Q}$ are replaced by $\bar{d}$ and $\bar{d}_{P Q}$.

To establish the Schauder estimates we first prove an interpolation inequality which is a parabolic version of those inequalities for elliptic problems stated in Section 6.8 of [3]. The method of proof here is also based on [3].

Lemma 4.4. Let $u \in C^{2+\alpha}(\mathcal{D})$. Then for any $\epsilon>0$ and some constant $C=$ $C(\epsilon)$ we have

$$
|d D u|_{\alpha}^{*} \leqslant C|u|_{0}+\epsilon|u|_{2+\alpha}^{*} .
$$

Proof: By the definition (4.15)

$$
|d D u|_{\alpha}^{*}=|d D u|_{0}+[d D u]_{\alpha}^{*} .
$$


We proceed to estimate $[d D u]_{\alpha}^{*}$ only, because Lemma 6.32 of $[3]$ can be applied to $|d D u|_{0}$ to obtain the bound

$$
|d D u|_{0} \leqslant C\left(\epsilon_{1}\right)|u|_{0}+\epsilon_{1}\left|d^{2} D^{2} u\right|_{0}
$$

for each $\epsilon_{1}$.

Let $P=(x, t), Q=(z, s) \in \mathcal{D}$. We define $r=\mu d_{P}$ and

$$
E_{r}=\left\{(\xi, \tau)|| \xi-x|<r,| \tau-t \mid<r^{2}\right\}
$$

where $0<\mu<1 / 2$ is a constant to be specified later. Let $P^{\prime}=\left(x^{\prime}, t\right), P^{\prime \prime}=\left(x^{\prime \prime}, t\right)$ be the end points of the line segment of length $2 r$ parallel to the $x_{i}$ axis and with its centre at $P$.

It is obvious that for $Q^{\prime}=(x, s)$

$$
\frac{|D u(P)-D u(Q)|}{d(P, Q)^{\alpha}} \leqslant \frac{\left|D u(P)-D u\left(Q^{\prime}\right)\right|}{d(P, Q)^{\alpha}}+\frac{\left|D u\left(Q^{\prime}\right)-D u(Q)\right|}{d(P, Q)^{\alpha}}
$$

By Lemma 6.32 of [3], we have, for each $\epsilon_{2}>0$

$$
d_{P Q}^{1+\alpha}\left|\frac{D u\left(Q^{\prime}\right)-D u(Q)}{d\left(Q^{\prime}, D\right)^{\alpha}}\right| \leqslant C\left(\epsilon_{2}\right)|u|_{0}+\epsilon_{2}\left|d^{2} D^{2} u\right|_{0}
$$

For the estimate of the first term on the right-hand side of (4.19), we consider

$$
w(x)=u(x, t)-u(x, s)
$$

By the theorem of the mean there is $\bar{x}$ on the segment between $x^{\prime}$ and $x^{\prime \prime}$ in $\Omega$ such that

$$
D_{i} w(\bar{x})=\frac{w\left(x^{\prime}\right)-w\left(x^{\prime \prime}\right)}{2 r}
$$

that is,

$$
D_{i} u(\bar{x}, t)-D_{i} u(\bar{x}, s)=\frac{1}{2 r}\left[\left(u\left(x^{\prime}, t\right)-u\left(x^{\prime}, s\right)\right)-\left(u\left(x^{\prime \prime}, t\right)-u\left(x^{\prime \prime}, s\right)\right)\right]
$$

Therefore, if $Q^{\prime} \in E_{r}$,

$$
D_{i} u(P)-D_{i} u(Q)=D_{i} u(\bar{x}, t)-D_{i} u(\bar{x}, s)+\int_{\bar{x}}^{x}\left[D_{i i} u(x, t)-D_{i i} u(x, s)\right] d x_{i}
$$


and hence

(4.22)

$$
\begin{aligned}
d_{P Q}^{1+\alpha} \frac{\left|D_{i} u(P)-D_{i} u(Q)\right|}{d(P, Q)^{\alpha}} \leqslant & \frac{d_{P Q}^{1+\alpha}}{2 r}\left(\frac{\left|\left(u\left(x^{\prime}, t\right)-u\left(x^{\prime}, s\right)\right)\right|}{d\left(P, Q^{\prime}\right)^{\alpha}}+\frac{\left|\left(u\left(x^{\prime \prime}, t\right)-u\left(x^{\prime \prime}, s\right)\right)\right|}{d\left(P, Q^{\prime}\right)^{\alpha}}\right) \\
& +d_{P Q}^{1+\alpha} \int_{\bar{x}}^{x} \frac{\left|D_{i i} u(x, t)-D_{i i} u(x, s)\right|}{d(P, Q)^{\alpha}} d x_{i} \\
& \leqslant \frac{d_{P Q}^{\alpha-1}\left|d^{2} D_{t} u\right|_{0} r^{2-\alpha}}{r}+d_{P Q}^{\alpha-1}\left[d^{2} D^{2} u\right]_{\alpha}^{*} r \\
& \leqslant \mu^{1-\alpha}\left|d^{2} D_{t} u\right|_{0}+\mu\left[d^{2} D^{2} u\right]_{\alpha}^{*}
\end{aligned}
$$

provided $d_{P Q}<1$.

If $Q^{\prime} \notin E_{r}$,

$$
d_{P Q}^{1+\alpha} \frac{\left|D u(P)-D u\left(Q^{\prime}\right)\right|}{d(P, Q)^{\alpha}} \leqslant \frac{2}{\mu^{\alpha}}|d D u|_{0}
$$

Inserting (4.18) into (4.23), we obtain

$$
d_{P Q}^{1+\alpha} \frac{\left|D u(P)-D u\left(Q^{\prime}\right)\right|}{d(P, Q)^{\alpha}} \leqslant \frac{2 C\left(\epsilon_{1}\right)}{\mu^{\alpha}}|u|_{0}+\frac{2 \epsilon_{1}}{\mu^{\alpha}}\left|d^{2} D^{2} u\right|_{0} .
$$

Now we choose $\mu, \epsilon_{2}$ such that $\mu^{1-\alpha}<\epsilon$ and $\mu, \epsilon_{2}<\epsilon$, and then choose $\epsilon_{1}$ such that $\epsilon_{1}<\epsilon$ and $\epsilon_{1} / \mu^{\alpha}<\epsilon$. Combining all the estimates above we obtain

$$
\begin{aligned}
{[d D u]_{\alpha}^{*} } & \leqslant C|u|_{0}+\epsilon\left|d D^{2} u\right|_{0}^{*}+\epsilon\left|d^{2} D_{t} u\right|_{0}^{*}+\epsilon\left[d^{2} D^{2} u\right]_{\alpha}^{*} \\
& \leqslant C|u|_{0}+\epsilon|u|_{2+\alpha}^{*} .
\end{aligned}
$$

LEMMA 4.5. Let $\Gamma$ be a portion of $\bar{\Omega}+S$ and $u \in C^{2+\alpha}(\mathcal{D} \cup \Gamma)$. Then for each $\epsilon>0$ and some constant $C=C(\epsilon)$, we have

$$
|d D u|_{\alpha, \mathcal{D} \cup \Gamma}^{*} \leqslant C|u|_{0}+\epsilon|u|_{2+\alpha, D \cup \Gamma}^{*}
$$

Proof: The proof of this lemma differs from that of Lemma 4.4 only at the choice of $P^{\prime}, P^{\prime \prime}$.

More precisely, assume first $\Gamma$ is only a flat portion of $S$, that is,

$$
\Gamma=\Sigma \times\left(t_{1}, t_{2}\right)
$$

where $\Sigma$ is a domain in $\mathbb{R}^{n-1}$ and $0<t_{1}<t_{2}<T$. 
To estimate $[d D u]_{\alpha}^{*}$ in such a case, we consider the line through $P$ parallel to the $x_{n}$ axis, and let $P^{\prime}, P^{\prime \prime}$ be the end points of the segment of the above line truncated by $\partial\left(E_{r} \cap \mathcal{D}\right)$. Then, instead of (4.19) we have

$$
D_{n} w(\bar{x})=\frac{w\left(x^{\prime}\right)-w\left(x^{\prime \prime}\right)}{d\left(P^{\prime}, P^{\prime \prime}\right)}
$$

where $d\left(P^{\prime}, P^{\prime \prime}\right) \geqslant r$.

The remaining proof is the same as before.

If $\Gamma$ is a portion of $\Omega$ only, the whole proof of Lemma 4.4 carries over. For general $\Gamma$, the result is only a combination of the two cases above.

The following Lemma is an immediate consequence of Lemmas 4.4 and 4.5.

LEMMA 4.6. If $u \in C^{2+\alpha}(\overline{\mathcal{D}})$ and $\partial \Omega$ is also of $C^{2+\alpha}$, then for each $\epsilon>0$ and some $C=C(\epsilon)$ we have

$$
|D u|_{\alpha} \leqslant|C u|_{0}+\epsilon|u|_{2+\alpha}
$$

Theorem 4.7. Let $\mathcal{D}$ be a $C^{2+\alpha}$ domain and $u \in C^{2+\alpha}(\mathcal{D})$ be a solution of (2.7)-(2.9), $f, g \in C^{\infty}(\overline{\mathcal{D}})$. Suppose that following conditions hold,

(a) $\quad a^{i j} \xi_{i} \xi_{j} \geqslant \lambda|\xi|^{2} \quad \forall \xi \in \mathbb{R}^{n}$

$\alpha^{i j} \eta_{i} \eta_{j} \geqslant \lambda|\eta|^{2} \quad \forall \eta \in \mathbb{R}^{n}$ such that $\eta \perp \nu$ and $(x, t) \in S$;

(b) $\left|a^{i j}, b^{i}, c\right|_{\alpha ; \mathcal{D}},\left|\alpha^{i j}, \beta^{i}, \gamma\right|_{\alpha ; \overline{\mathcal{D}}} \leqslant \Lambda$ for some $\Lambda>0$.

We then have

$$
|u|_{2+\alpha: \mathcal{D}} \leqslant C\left(|u|_{0 ; \mathcal{D}}+|\varphi|_{2+\alpha ; \mathcal{D}}+|g|_{\alpha ; \mathcal{D}}+|f|_{\alpha ; \mathcal{D}}\right)
$$

where $C=C(n, \alpha, \lambda, \Lambda, \epsilon, S)$.

Proof: We assume $u \in C^{2+\alpha}$ is a solution of equations (2.7)-(2.9) and suppose that the boundary is locally flattened, that is (2.9) becomes

$$
\alpha^{s t} D_{s t} u+\beta^{i} D_{i} u+\gamma u-D_{t} u=g \quad \text { on } B^{2} \times(0, T) .
$$

Setting $G=g-\beta^{n} D_{n} u$, we can rewrite (4.30) as

$$
\alpha^{s t} D_{s t} u+\beta^{i} D_{i} u+\gamma u-D_{t} u=G \quad \text { on } B^{0} \times(0, T)
$$

Thus, (4.31) may be considered as a parabolic equation in the $n$ dimensional domain $B^{0} \times(0, T)$. If we apply the Schauder interior estimates of the first initial-boundary value problem [2] for this equation, we can obtain for $G \in C^{\alpha}\left(B^{0} \times(0, T)\right)$,

$$
|u|_{2+\alpha ; B^{0}}^{*} \leqslant C\left(|u|_{0 ; B^{0}}+\left|d^{2} G\right|_{\alpha ; B^{0}}^{*}\right)
$$


where $C=C(n, \alpha, \lambda, \Lambda)$. This implies that

$$
|u|_{2+\alpha ; S} \leqslant C\left(|u|_{0 ; \mathcal{D}}+|g|_{\alpha ; \mathcal{D}}+C_{1}|D u|_{\alpha ; \mathcal{D}}\right) \text {. }
$$

Using the global estimates of the first initial-boundary value problem Theorem 3.2 .6 of [2], we have

$$
|u|_{2+\alpha ; \mathcal{D}} \leqslant C\left(|u|_{0 ; \mathcal{D}}+|u|_{2+\alpha ; S}+|\varphi|_{2+\alpha ; \mathcal{D}}+|f|_{\alpha ; \mathcal{D}}\right) \text {. }
$$

Now, we insert (4.32) into (4.33) to obtain,

$$
|u|_{2+\alpha ; \mathcal{D}} \leqslant C\left(|u|_{0 ; \mathcal{D}}+|u|_{0 ; \mathcal{D}}+|D u|_{\alpha ; \mathcal{D}}+|\varphi|_{2+\alpha ; \mathcal{D}}+|g|_{\alpha ; \mathcal{D}}+|f|_{\alpha ; \mathcal{D}}\right)
$$

where $C=C(n, \alpha, \lambda, \Lambda, S)$. We have by Lemma 4.6,

$$
|D u|_{\alpha ; \mathcal{D}} \leqslant C_{2}|u|_{0 ; \mathcal{D}}+\epsilon|u|_{2+\alpha ; \mathcal{D}}
$$

If we substitute the right-hand side of (4.36) into (4.35), we obtain

$$
|u|_{2+\alpha ; \mathcal{D}} \leqslant C\left(|u|_{0 ; \mathcal{D}}+C_{2}|u|_{0 ; \mathcal{D}}+\epsilon|u|_{2+\alpha ; \mathcal{D}}+|\varphi|_{2+\alpha ; \mathcal{D}}+|g|_{\alpha ; \mathcal{D}}+|f|_{\alpha ; \mathcal{D}}\right) \text {. }
$$

Hence, we have, for sufficiently small $\epsilon$

$$
|u|_{2+\alpha ; \mathcal{D}} \leqslant C\left(|u|_{0 ; \mathcal{D}}+|\varphi|_{2+\alpha ; \mathcal{D}}+|g|_{\alpha ; \mathcal{D}}+|f|_{\alpha ; \mathcal{D}}\right)
$$

where $C=C(n, \alpha, \lambda, \Lambda, S)$.

\section{REFERENCES}

[1] J.R. Cannon and G.H. Meyer, 'On diffusion in a fractured medium', SIAM J. Appl. Math. 3 (1971), 434-448.

[2] A. Friedman, Partial differential equations of parabolic type (Prentice-Hall, Inc., Englewood Cliffs NJ, London, 1964).

[3] D. Gilbarg and N.S. Trudinger, Elliptic partial differential equations of second order, Second Edition (Springer-Verlag, Berlin, Heidelberg, New York, Tokyo, 1983).

[4] P. Korman, 'Existence of solutions for a class of semilinear noncoercive problems', Nonlinear Anal. 10 (1986), 1471-1476.

[5] Y. Luo and N.S. Trudinger, 'Linear second order elliptic equations with Venttsel boundary conditions', Proc. Roy. Soc. Edinburgh Sect. A 118 (1991), 193-270.

[6] Y. Luo, 'On the quasilinear elliptic Venttsel boundary value problem', Nonlinear Anal. 16 (1991), 761-769.

Department of Mathematics

Royal Melbourne Institute of Technology

GPO Box $2476 \mathrm{~V}$

Melbourne, Vic. 3001

Australia 